\title{
Skin Hypopigmentation, CTCAE
}

National Cancer Institute

\section{Source}

National Cancer Institute. Skin Hypopigmentation, CT CAE. NCI Thesaurus. Code

C143836.

A disorder characterized by loss of skin pigment (e.g., vitiligo). 